PROCEEDINGS OF THE

AMERICAN MATHEMATICAL SOCIETY

Volume 132, Number 9, Pages 2639-2646

S 0002-9939(04)07452-0

Article electronically published on March 25, 2004

\title{
A JOINT THEOREM GENERALIZING THE CRITERIA OF MONTEL AND MIRANDA FOR NORMAL FAMILIES
}

\author{
BAO QIN LI \\ (Communicated by Mei-Chi Shaw)
}

\begin{abstract}
We give a joint normality theorem, which simultaneously generalizes Montel's criterion and Miranda's criterion for normal families.
\end{abstract}

\section{$\S 1$. INTRODUCTION}

We will give in this paper a joint theorem on normal families of holomorphic functions in a domain $D$ of the complex plane, which simultaneously generalizes the well-known Montel's criterion and Miranda's criterion of normality.

Perhaps the most celebrated theorem in the theory of normal families is the following criterion of Montel, who created the theory of normal families.

Theorem A (Montel's Criterion $[\mathrm{MO}]$ ). Let $\mathcal{F}$ be a family of holomorphic functions in a domain $D$ of the complex plane. If for all $f \in \mathcal{F}, f \neq 0$ and $f \neq 1$, then $\mathcal{F}$ is a normal family.

As is well known, Montel's theorem has important consequences such as the Big Picard Theorem and Julia's theorem on the existence of a Julia direction for entire functions. The meromorphic version of Montel's theorem, requiring that $f$ omits three distinct points $a, b, c$ on the Riemann sphere, follows immediately from the above theorem, by simply setting $g=\frac{1}{f-a}$, which is then entire (assuming that $a \neq \infty)$. Montel's theorem is called the Fundamental Normality Test in the book $[$ ]. The notion of normal family plays an important role in complex function theory and has a wide range of applications to complex dynamics, extremal problems, etc. (cf. $[\mathbf{F}],[S]$, etc.).

Another well-known result on normal families of holomorphic functions is the following Miranda's criterion.

Theorem B (Miranda's Criterion [Mi]). Let $\mathcal{F}$ be a family of holomorphic functions in a domain $D$ of the complex plane. If for all $f \in \mathcal{F}, f \neq 0$ and $f^{\prime} \neq 1$, then $\mathcal{F}$ is a normal family.

Received by the editors June 3, 2003

2000 Mathematics Subject Classification. Primary 30D45, 30D35.

Key words and phrases. Holomorphic function, normal family, Nevanlinna theory, order, spherical derivative.

Supported in part by NSF Grant DMS-0100486. 
In the previous theorem, $f^{\prime}$ can be replaced by a higher-order derivative of $f$. There have been various generalizations of Montel's criterion and Miranda's criterion in the literature. In particular, Pang recently obtained the following extension of Miranda's criterion.

Theorem $\mathbf{C}([\mathrm{P})$. Let $\mathcal{F}$ be a family of holomorphic functions in a domain $D$ of the complex plane, $a \neq 0$ a complex number, and $B$ a positive constant. Suppose that for all $f \in \mathcal{F}$,

(a) $f^{\prime}(z)=a$ whenever $z \in f^{-1}(0)$; and

(b) $\left|f^{\prime \prime}(z)\right| \leq B$ whenever $z \in\left(f^{\prime}-a\right)^{-1}(0)$.

Then $\mathcal{F}$ is a normal family.

Here and in the following, as usual, we use $g^{-1}(0)$ to denote the zero set of $g$, where $g$ is a function or $g=\left(g_{1}, \ldots, g_{m}\right)$, a map defined in a domain $D \subseteq \mathbf{C}^{n}$. That is, $g^{-1}(0)=\left\{z \in D: g_{1}(z)=\cdots=g_{m}(z)=0\right\}$. Theorem $\mathrm{C}$ reduces to Theorem B when $f(z) \neq 0$ and $f^{\prime}(z) \neq a$ in $D$ for every $f \in \mathcal{F}$, in which case $f^{-1}(0)=\left(f^{\prime}-a\right)^{-1}(0)=\emptyset$ and thus the conditions (a) and (b) hold vacuously. The conditions of Theorem $\mathrm{C}$ require that every zero of $f$ must be an $a$-point of $f^{\prime}$, and $f^{\prime \prime}$ is bounded on the set $\left(f^{\prime}-a\right)^{-1}(0)$ and thus also on the set $f^{-1}(0)$, since $f^{-1}(0) \subseteq\left(f^{\prime}-a\right)^{-1}(0)$.

In the present paper, we obtain the following joint theorem, which simultaneously generalizes both Montel's criterion and Miranda's criterion, and in fact includes all the previous three theorems as simple consequences.

Theorem 1.1. Let $\mathcal{F}$ be a family of holomorphic functions in a domain $D$ of the complex plane, $a, b$ nonzero complex numbers, and $A, B$ positive constants. Suppose that for all $f, g \in \mathcal{F}$,

(i) $A \leq\left|f^{\prime}(z)\right| \leq B$ and $\left|f^{\prime \prime}(z)\right| \leq B$ whenever $z \in f^{-1}(0)$; and

(ii) $\min \left\{\left|f^{\prime \prime}\left(z_{1}\right)\right|,\left|g^{\prime}\left(z_{2}\right)\right|\right\} \leq B$ whenever $\left(z_{1}, z_{2}\right) \in F^{-1}(0)$, where $F\left(z_{1}, z_{2}\right):=$ $\left(f^{\prime}\left(z_{1}\right)-a, g\left(z_{2}\right)-b\right)$.

Then $\mathcal{F}$ is a normal family.

It is trivial to note that the bound on $\left|f^{\prime \prime}(z)\right|$ in (i) and the bound on $\min \left\{\left|f^{\prime \prime}\left(z_{1}\right)\right|,\left|g^{\prime}\left(z_{2}\right)\right|\right\}$ in (ii) can be different from $B$.

Remark 1.2. (1) If $f \neq 0$ and $f \neq b$ for every $f \in \mathcal{F}$ in Theorem 1.1, then $f^{-1}(0)=\emptyset$ and $F^{-1}(0)=\emptyset$, and thus the conditions (i) and (ii) in Theorem 1.1 hold vacuously. Therefore, Theorem 1.1 extends Montel's criterion.

(2) If $f \neq 0$ and $f^{\prime} \neq a$ for every $f \in \mathcal{F}$, then the conditions (i) and (ii) in Theorem 1.1 also hold vacuously. Thus, Theorem 1.1 also extends Miranda's criterion.

(3) The condition (a) of Theorem $\mathrm{C}$, which requires that $f^{\prime}(z)=a$ (and thus $\left.\left|f^{\prime}(z)\right|=|a|\right)$ whenever $f(z)=0$, is fairly strong, and is clearly stronger than the boundedness condition on $f^{\prime}$ in Theorem 1.1 (i). As mentioned earlier, the condition that $\left|f^{\prime \prime}(z)\right| \leq B$ on $f^{-1}(0)$ is implicit in Theorem $\mathrm{C}$.

If the condition (b) in Theorem $\mathrm{C}$ holds, i.e., $\left|f^{\prime \prime}(z)\right| \leq B$ whenever $f^{\prime}(z)=a$ for every $f \in \mathcal{F}$, then for every $\left(z_{1}, z_{2}\right) \in F^{-1}(0)$, where $F=\left(f^{\prime}\left(z_{1}\right)-a, g\left(z_{2}\right)-b\right)$, we have that $f^{\prime}\left(z_{1}\right)=a$ and thus that $\left|f^{\prime \prime}\left(z_{1}\right)\right| \leq B$. Therefore, $\min \left\{\left|f^{\prime \prime}\left(z_{1}\right)\right|,\left|g^{\prime}\left(z_{2}\right)\right|\right\}$ $\leq B$. This shows that the condition (b) in Theorem $\mathrm{C}$ implies and thus is stronger than the condition (ii) in Theorem 1.1. Thus, we see that Theorem 1.1 also extends Theorem C. 
By way of illustration, we give a simple normal family that satisfies the conditions of Theorem 1.1, but not the conditions of Theorem $\mathrm{C}$ (Theorem A and Theorem B). Consider $\left\{f_{n}\right\}=\left\{\frac{1}{n} \sin n z\right\}$ in the unit disc. Then $f_{n}^{\prime}(z)=\cos n z$ and $f_{n}^{\prime \prime}(z)=$ $-n \sin n z$ for each $f_{n}$ in the family. When $f_{n}(z)=0, n z=k \pi$ ( $k$ is an integer), and thus the condition (i) of Theorem 1.1 is trivially satisfied. When $f_{n}^{\prime}(z)=1$, we have that $f^{\prime \prime}(z)=\sin n z=0$ and thus the condition (ii) of Theorem 1.1 is also trivially satisfied. However, it is easy to see that for any complex number $a$ there exist infinitely many $n$ 's such that at the point $\frac{\pi}{4}$ in the unit disc, $f_{n}\left(\frac{\pi}{4}\right)=0$, but $f_{n}^{\prime}\left(\frac{\pi}{4}\right) \neq a$. Thus, this family does not satisfy the conditions in Theorem C.

Related results on normal families in several complex variables can be found in L].

\section{$\S 2$. Proof of TheOrem 1.1}

In proving the theorem, we will employ Nevanlinna theory and will assume familiarity with standard notation in the theory (see e.g. [H]) such as counting functions $\bar{N}\left(r, \frac{1}{g-c}\right)$ for the zeros of $g-c$ (without counting multiplicities) and $N\left(r, \frac{1}{g-c}\right)$ (counting multiplicities), the Nevanlinna characteristic $T(r, g)$, and the "error" term $S(r, g)$, which denotes a quantity satisfying $S(r, g)=o\{T(r, g\}$ outside possibly a set $r$ of finite Lebesgue measure. We will also need the following lemma in $[\mathrm{P}]$.

Lemma 2.1. Let $\mathcal{F}$ be a family of holomorphic functions that is not normal in the unit disc. Suppose that there exists a positive number $B$ such that $\left|f^{\prime}(z)\right| \leq B$ whenever $f \in \mathcal{F}$ and $f(z)=0$. Then for any number $0 \leq k \leq 1$ there exist

(i) a positive number $r, 0<r<1$;

(ii) complex numbers $z_{n},\left|z_{n}\right|<r$;

(iii) functions $f_{n} \in \mathcal{F}$;

(iv) positive numbers $a_{n}, \lim _{n \rightarrow \infty} a_{n}=0$, such that $g_{n}(z)=\frac{f_{n}\left(z_{n}+a_{n} z\right)}{a_{n}^{k}}$ converges locally uniformly (with respect to the spherical metric) on compact sets to a nonconstant entire function $G$ in $\mathbf{C}$ such that $G^{\#}(z) \leq G^{\#}(0)=B+1$ and (thus) $\operatorname{Ord}(G) \leq 1$.

Here, $G^{\#}(z):=\frac{\left|G^{\prime}(z)\right|}{1+|G(z)|^{2}}$ is the spherical derivative of $G$ at $z$ and $\operatorname{Ord}(G)$ is the order of $G$. The above lemma is an infinitesimal form of the classic Marty inequality and is a generalization of Zalcman's lemma $[\mathrm{Z}]$. That the order of $G$ is at most one is a result of Clunie-Hayman $[\mathrm{CH}]$, which states that the order of an entire function with uniformly bounded spherical derivative on $\mathbf{C}$ is at most one.

Proof of Theorem 1.1. Since normality is a local property, it is easy to see that we may assume that the domain $D$ is the unit disc, using the transformation $z=$ $Z_{0}+\epsilon Z(|Z| \leq 1)$ if necessary, where $Z_{0} \in D$ is an arbitrarily given point and $\epsilon>0$ is a positive number so that $\left\{z:\left|z-Z_{0}\right| \leq \epsilon\right\} \subset D$. (It is worth noting here that the conditions of the theorem are not invariant under this transformation, which, however, does not cause any problems, since $a$ and $b$ in the theorem are allowed to be different.) Suppose, to the contrary, that $\mathcal{F}$ is not normal. Our goal is to obtain a contradiction in the sequel. Given $0 \leq k \leq 1$, we obtain from the above lemma,

(i) a positive number $r, 0<r<1$;

(ii) complex numbers $z_{n, k},\left|z_{n, k}\right|<r$;

(iii) functions $f_{n, k} \in \mathcal{F}$; 
(iv) positive numbers $a_{n, k}, \lim _{n \rightarrow \infty} a_{n, k}=0$, such that $g_{n, k}(z)=\frac{f_{n, k}\left(z_{n, k}+a_{n, k} z\right)}{a_{n, k}^{k}}$ converges locally uniformly (with respect to the spherical metric) on compact sets to a non-constant entire function $G_{k}$ in $\mathbf{C}$ such that $G_{k}^{\#}(z) \leq G_{k}^{\#}(0)=B+1$ and $\operatorname{Ord}\left(G_{k}\right) \leq 1$. We denote $G_{1}$ by $u$, and $G_{0}$ by $v$ in the following proof. That is,

$$
u(z)=\lim _{n \rightarrow \infty} g_{n, 1}(z)=\lim _{n \rightarrow \infty} \frac{f_{n, 1}\left(z_{n, 1}+a_{n, 1} z\right)}{a_{n, 1}}
$$

and

$$
v(z)=\lim _{n \rightarrow \infty} g_{n, 0}(z)=\lim _{n \rightarrow \infty} f_{n, 0}\left(z_{n, 0}+a_{n, 0} z\right) .
$$

We first prove the following

Claim 1. $A \leq\left|u^{\prime}(\zeta)\right| \leq B, u^{\prime \prime}(\zeta)=0$ whenever $\zeta \in u^{-1}(0)$. Also, $v^{\prime}(\zeta)=v^{\prime \prime}(\zeta)=0$ whenever $\zeta \in v^{-1}(0)$.

Let $\zeta \in u^{-1}(0)$. Then $u(\zeta)=0$. Since $u \neq \equiv$, there exists a $0<\rho<1$ such that $u(z) \neq 0$ on $|z-\zeta|=\rho$. By Hurwitz's theorem, for large $n, g_{n, 1}$ and $u$ have the same number of zeros on $|z-\zeta|<\rho$. Thus, there exist $\zeta_{n}$ with $\left|\zeta_{n}-\zeta\right|<\rho$ such that $g_{n, 1}\left(\zeta_{n}\right)=0$, which implies that $f_{n, 1}\left(z_{n, 1}+a_{n, 1} \zeta_{n}\right)=0$. By the hypotheses of the theorem,

$$
A \leq\left|f_{n, 1}^{\prime}\left(z_{n, 1}+a_{n, 1} \zeta_{n}\right)\right| \leq B, \quad\left|f_{n, 1}^{\prime \prime}\left(z_{n, 1}+a_{n, 1} \zeta_{n}\right)\right| \leq B .
$$

However, $g_{n, 1}^{\prime}(z)=f_{n, 1}^{\prime}\left(z_{n, 1}+a_{n, 1} z\right)$. We thus have that $A \leq\left|g_{n, 1}^{\prime}\left(\zeta_{n}\right)\right| \leq B$. But, $u^{\prime}(z)=\lim _{n \rightarrow \infty} g_{n, 1}^{\prime}(z)$. Hence, $A \leq\left|u^{\prime}(\zeta)\right| \leq B$. We also have that

$$
u^{\prime \prime}(\zeta)=\lim _{n \rightarrow \infty} g_{n, 1}^{\prime \prime}\left(\zeta_{n}\right)=\lim _{n \rightarrow \infty} a_{n, 1} f_{n, 1}^{\prime \prime}\left(z_{n, 1}+a_{n, 1} \zeta_{n}\right)=0 .
$$

Using the definition of $v$ and the same argument as above, we can show that $v^{\prime}(\zeta)=v^{\prime \prime}(\zeta)=0$ whenever $\zeta \in v^{-1}(0)$. This proves the claim. We next prove

Claim 2. The function $u$ is nonlinear, and each of the equations $u^{\prime}(z)=a$ and $v(z)=b$ has at least one solution.

If $u$ is linear, then $u^{\prime}$ is a constant, and there exists a complex number $\eta$ such that $u(\eta)=0$, since $u$ is nonconstant. By Claim $1,\left|u^{\prime}(\eta)\right| \leq B$. Thus, $u^{\prime}(0)=u^{\prime}(\eta) \leq B$, and then $B+1=u^{\#}(0) \leq u^{\prime}(0) \leq B$, which is absurd. This shows that $u$ is nonlinear.

If $u^{\prime}(z)=a$ has no solutions, then $u^{\prime}(z)=a+\alpha e^{\beta z}$ with $\alpha \beta \neq 0$ in view of the fact that $u$ is nonlinear and the order of $u$ and thus $u^{\prime}$ is at most one. We then have that $u(z)=a z+\frac{\alpha}{\beta} e^{\beta z}$, which has zeros, and $u^{\prime \prime}(z)=\alpha \beta e^{\beta z} \neq 0$, which does not have any zeros. Consider a $\zeta$ such that $u(\zeta)=0$. Then $u^{\prime \prime}(\zeta) \neq 0$, which contradicts Claim 1.

The same proof works for the equation $v(z)=b$, which must have solutions, since otherwise $v(z)=b+\alpha e^{\beta z}$ with $\alpha \beta \neq 0$ and thus $v^{\prime}(z)=\alpha \beta e^{\beta z}$. Then $v(z)=0$ has solutions, but $v^{\prime}(z)=0$ does not have any solutions, which again contradicts Claim 1.

We now return to the proof of the theorem. By Claim $2, u^{\prime}(z)=a$ and $v(z)=b$ both have solutions. Suppose that $z_{0}$ is an arbitrary solution of $v(z)=b$ and $w_{0}$ is an arbitrary solution of $u^{\prime}(z)=a$. Since $v \not \equiv b$ and $g_{n, 0}(z) \rightarrow v(z)$, by Hurwitz's theorem there exist $z_{n} \rightarrow z_{0}$ such that $g_{n, 0}\left(z_{n}\right)=b$, i.e., $f_{n, 0}\left(z_{n, 0}+a_{n, 0} z_{n}\right)=b$ for large $n$. Similarly, noting that $u^{\prime} \not \equiv a$ by Claim 2 and $g_{n, 1}^{\prime}(z) \rightarrow u^{\prime}(z)$, we see that 
there exist $w_{n} \rightarrow w_{0}$ such that $g_{n, 1}^{\prime}\left(w_{n}\right)=a$, i.e., $f_{n, 1}^{\prime}\left(z_{n, 1}+a_{n, 1} w_{n}\right)=a$ for large $n$. Thus,

$$
\left(z_{n, 1}+a_{n, 1} w_{n}, z_{n, 0}+a_{n, 0} z_{n}\right) \in F^{-1}(0),
$$

where $F\left(z_{1}, z_{2}\right):=\left(f_{n, 1}^{\prime}\left(z_{1}\right)-a, f_{n, 0}\left(z_{2}\right)-b\right)$ for large $n$. By the assumption (ii) of the theorem, we have that

$$
\min \left\{\left|f_{n, 1}^{\prime \prime}\left(z_{n, 1}+a_{n, 1} w_{n}\right)\right|,\left|f_{n, 0}^{\prime}\left(z_{n, 0}+a_{n, 0} z_{n}\right)\right|\right\} \leq B .
$$

We next prove that it is impossible to have infinitely many $n$ 's such that

$$
\min \left\{\left|f_{n, 1}^{\prime \prime}\left(z_{n, 1}+a_{n, 1} w_{n}\right)\right|,\left|f_{n, 0}^{\prime}\left(z_{n, 0}+a_{n, 0} z_{n}\right)\right|\right\}=\left|f_{n, 1}^{\prime \prime}\left(z_{n, 1}+a_{n, 1} w_{n}\right)\right| .
$$

Suppose that (2.2) held for infinitely many $n$ 's; we would then have, by passing to a subsequence and in view of (2.1), that

$$
u^{\prime \prime}\left(w_{0}\right)=\lim _{n \rightarrow \infty} g_{n, 1}^{\prime \prime}\left(w_{n}\right)=\lim _{n \rightarrow \infty} a_{n, 1} f_{n, 1}^{\prime \prime}\left(z_{n, 1}+a_{n, 1} w_{n}\right)=0 .
$$

Thus, we would have a $w_{0}$ satisfying $u^{\prime}\left(w_{0}\right)=a$ and $u^{\prime \prime}\left(w_{0}\right)=0$. A contradiction will be obtained after we prove the following

Claim 3. If there exists a $w_{0}$ such that $u^{\prime}\left(w_{0}\right)=a$ and $u^{\prime \prime}\left(w_{0}\right)=0$, then $\left(u^{2}-a^{2}\right)^{-1}(0) \subseteq\left(u^{\prime \prime}\right)^{-1}(0)$.

To prove the claim, we first notice that the function $\frac{u^{\prime \prime}}{u}$ is entire, since, by Claim 1 , every zero of $u$ is simple and is also a zero of $u^{\prime \prime}$. Recall that the order of $u$ is at most 1. Thus, by Hadamard's Factorization Theorem (see e.g. [B]), we have that $u(z)=G e^{\gamma z+\delta}$, where $\gamma$ and $\delta$ are complex numbers and

$$
G=z^{m} \Pi_{n \geq 1}\left(1-\frac{z}{\gamma_{n}}\right) e^{\frac{z}{\gamma_{n}}}
$$

is the canonical product for the zeros $\gamma_{n}$ of $u$ and $m$ is a nonnegative integer (actually, $m=0$ or 1 since $u$ has only simple zeros). We then have that

$$
\frac{u^{\prime \prime}}{u}=\frac{G^{\prime \prime}}{G}+2 \gamma \frac{G^{\prime}}{G}+\gamma^{2} .
$$

Note that $\left(\frac{G^{\prime}}{G}\right)^{\prime}=\frac{G^{\prime \prime} G-G^{\prime} 2}{G^{2}}$ or $\frac{G^{\prime \prime}}{G}=\left(\frac{G^{\prime}}{G}\right)^{\prime}+\left(\frac{G^{\prime}}{G}\right)^{2}$. Thus,

Also, note that

$$
\frac{u^{\prime \prime}}{u}=\left(\frac{G^{\prime}}{G}\right)^{\prime}+\left(\frac{G^{\prime}}{G}\right)^{2}+2 \gamma \frac{G^{\prime}}{G}+\gamma^{2} .
$$

$$
\frac{G^{\prime}}{G}=\frac{m}{z}+\sum_{n \geq 1}\left(\frac{1}{z-\gamma_{n}}+\frac{1}{\gamma_{n}}\right)
$$

Thus, we have that

$$
\begin{aligned}
\frac{u^{\prime \prime}}{u}=- & \frac{m}{z^{2}}-\sum_{n \geq 1} \frac{1}{\left(z-\gamma_{n}\right)^{2}}+\left(\frac{m}{z}+\sum_{n \geq 1}\left(\frac{1}{z-\gamma_{n}}+\frac{1}{\gamma_{n}}\right)\right)^{2}+2 \gamma \frac{m}{z} \\
& +2 \gamma \sum_{n \geq 1}\left(\frac{1}{z-\gamma_{n}}+\frac{1}{\gamma_{n}}\right)+\gamma^{2} .
\end{aligned}
$$

The terms of the series on the right-hand side of the above identity are functions in $\frac{1}{z-\gamma_{n}}(n \geq 1)$. After combining the like terms, one can see that the right-hand side must be a constant, since otherwise the right-hand side would have poles, which contradicts the fact that $\frac{u^{\prime \prime}}{u}$ is entire. Thus, we can write $u^{\prime \prime}=c^{2} u$, where $c$ is a constant, which is nonzero since $u$ is nonlinear. Solving this differential equation, we obtain that $u(z)=c_{1} e^{c z}+c_{2} e^{-c z}$, where $c_{1}$ and $c_{2}$ are two constants, none of 
which is zero since $u$ is non-constant and $u^{\prime \prime}$ (and thus $u$ ) has a zero $w_{0}$. From the equalities $u\left(w_{0}\right)=0$ and $u^{\prime}\left(w_{0}\right)=a$, we have that

$$
c_{1} e^{c w_{0}}+c_{2} e^{-c w_{0}}=0, \quad c c_{1} e^{c w_{0}}-c c_{2} e^{-c w_{0}}=a .
$$

We can view the above two equalities as a system in $e^{c w_{0}}$ and $e^{-c w_{0}}$. Solving this system, we obtain that $e^{c w_{0}}=\frac{a}{2 c c_{1}}$ and $e^{-c w_{0}}=\frac{-a}{2 c c_{2}}$, which implies that

or

$$
1=e^{c w_{0}} e^{-c w_{0}}=\frac{-a^{2}}{4 c^{2} c_{1} c_{2}}
$$

$$
a^{2}+4 c^{2} c_{1} c_{2}=0 .
$$

Now suppose that $w$ is an arbitrary zero (if there is any) of $u^{\prime}(w)-a$ or $u^{\prime}(w)+a$. That is,

$$
c c_{1} e^{c w}-c c_{2} e^{-c w}= \pm a \quad \text { or } \quad c c_{1} e^{2 c w} \mp a e^{c w}-c c_{2}=0 .
$$

Solving this quadratic equation in $e^{c w}$ yields that

$$
e^{c w}=\frac{ \pm a \pm \sqrt{a^{2}+4 c^{2} c_{1} c_{2}}}{2 c c_{1}}=\frac{ \pm a}{2 c c_{1}}
$$

in view of (2.3). We then obtain that

$$
u(w)=c_{1} e^{c w}+c_{2} e^{-c w}=c_{1} \frac{ \pm a}{2 c c_{1}}+c_{2} \frac{2 c c_{1}}{ \pm a}= \pm \frac{a^{2}+4 c^{2} c_{1} c_{2}}{2 c a}=0
$$

by (2.3) again. Thus, we have shown that every zero of $u^{\prime}-a$ and $u^{\prime}+a$ must be a zero of $u$ and thus $u^{\prime \prime}$, since $u^{\prime \prime}=c^{2} u$. This proves Claim 3 .

We can now use Nevanlinna theory to deduce a contradiction from (2.2). Recall the following Nevanlinna Second Fundamental Theorem $([\mathrm{N}],[\mathrm{H}])$ : For any nonconstant entire function $f$ and two distinct complex numbers $\alpha$ and $\beta$,

$$
T(r, f) \leq \bar{N}\left(r, \frac{1}{f-\alpha}\right)+\bar{N}\left(r, \frac{1}{f-\beta}\right)+S(r, f) .
$$

Applying this theorem to $u^{\prime}$, we obtain that

$$
T\left(r, u^{\prime}\right) \leq \bar{N}\left(r, \frac{1}{u^{\prime}-a}\right)+\bar{N}\left(r, \frac{1}{u^{\prime}+a}\right)+S\left(r, u^{\prime}\right) .
$$

By Claim 1, every zero of $u$ is simple and $u^{-1}(0) \subseteq\left(u^{\prime \prime}\right)^{-1}(0)$. Combining this fact with Claim 3, we deduce that

$$
\begin{aligned}
& N\left(r, \frac{1}{u}\right)+T\left(r, u^{\prime}\right) \\
& \leq \bar{N}\left(r, \frac{1}{u}\right)+\bar{N}\left(r, \frac{1}{u^{\prime}-a}\right)+\bar{N}\left(r, \frac{1}{u^{\prime}+a}\right)+S\left(r, u^{\prime}\right) \\
& \leq N\left(r, \frac{1}{u^{\prime \prime}}\right)+S\left(r, u^{\prime}\right) \leq T\left(r, u^{\prime \prime}\right)+S\left(r, u^{\prime}\right) \leq T\left(r, u^{\prime}\right)+S(r, u),
\end{aligned}
$$

in view of the fact that $T\left(r, h^{\prime}\right) \leq T(r, h)+S(r, h)$ for an entire function $h$. Thus, we have that $N\left(r, \frac{1}{u}\right)=S(r, u)$. We then use another well-known theorem, Milloux's Inequality, in Nevanlinna theory $([\overrightarrow{\mathrm{H}}])$ : For a non-constant entire function $f$ and a constant $\alpha \neq 0$,

$$
T(r, f) \leq N\left(r, \frac{1}{f}\right)+N\left(r, \frac{1}{f^{(k)}-\alpha}\right)-N\left(r, \frac{1}{f^{(k+1)}}\right)+S(r, f) .
$$


Applying this inequality twice to the function $u$, we obtain that

$$
T(r, u) \leq N\left(r, \frac{1}{u}\right)+N\left(r, \frac{1}{u^{\prime}-a}\right)-N\left(r, \frac{1}{u^{\prime \prime}}\right)+S(r, f)=\bar{N}\left(r, \frac{1}{u^{\prime}-a}\right)+S(r, u)
$$

and

$$
T(r, u) \leq N\left(r, \frac{1}{u}\right)+N\left(r, \frac{1}{u^{\prime}+a}\right)-N\left(r, \frac{1}{u^{\prime \prime}}\right)+S(r, f)=\bar{N}\left(r, \frac{1}{u^{\prime}+a}\right)+S(r, u) .
$$

Adding the above two inequalities yields that

$$
\begin{aligned}
& 2 T(r, u) \leq \bar{N}\left(r, \frac{1}{u^{\prime}-a}\right)+\bar{N}\left(r, \frac{1}{u^{\prime}+a}\right)+S(r, u) \\
& \leq N\left(r, \frac{1}{u^{\prime \prime}}\right)+S(r, u) \leq T\left(r, u^{\prime \prime}\right)+S(r, u) \leq T(r, u)+S(r, u),
\end{aligned}
$$

from which it follows that $T(r, u) \leq S(r, u)$ and thus that $u$ is a constant, a contradiction.

We have thus shown that (2.2) is impossible. Therefore, by (2.1), there must exist infinitely many positive integers $n$ such that

$$
\min \left\{\left|f_{n, 1}^{\prime \prime}\left(z_{n, 1}+a_{n, 1} w_{n}\right)\right|,\left|f_{n, 0}^{\prime}\left(z_{n, 0}+a_{n, 0} z_{n}\right)\right|\right\}=\left|f_{n, 0}^{\prime}\left(z_{n, 0}+a_{n, 0} z_{n}\right)\right| \leq B
$$

Hence, by passing to a subsequence, we obtain that

$$
v^{\prime}\left(z_{0}\right)=\lim _{n \rightarrow \infty} g_{n, 0}^{\prime}\left(z_{n}\right)=\lim _{n \rightarrow \infty} a_{n, 0} f_{n, 0}^{\prime}\left(z_{n, 0}+a_{n, 0} z_{n}\right)=0 .
$$

But, $z_{0}$ is an arbitrary zero of $v(z)-b$. We thus have shown that the multiplicity of an arbitrary zero of $v(z)-b$ is at least two. By Claim 1, we also know that the multiplicity of an arbitrary zero of $v(z)$ is at least three. We can then apply the Nevanlinna Second Fundamental Theorem again to obtain that

$$
\begin{aligned}
& T(r, v) \leq \bar{N}\left(r, \frac{1}{v}\right)+\bar{N}\left(r, \frac{1}{v-b}\right)+S(r, v) \\
& \leq \frac{1}{3} N\left(r, \frac{1}{v}\right)+\frac{1}{2} N\left(r, \frac{1}{v-b}\right)+S(r, v) \leq \frac{5}{6} T(r, v)+S(r, v)
\end{aligned}
$$

and thus that $T(r, v)=S(r, v)$, which implies that $v$ is a constant, a contradiction. This finally completes the proof.

\section{REFERENCES}

[B] C. A. Berenstein and R. Gay, Complex Variables, Springer-Verlag, New York, 1991. MR 92f:30001

$[\mathrm{CH}]$ J. Clunie and W. K. Hayman, The spherical derivative of integral and meromorphic functions, Comment. Math. Helv. 40 (1966), 117-148. MR 33:282

[F] J.-E. Fornaess, Dynamics in Several Complex Variables, CBMS Regional Conference Series in Mathematics, vol. 87, American Mathematical Society, Providence, RI, 1996. MR 96j:32033

[H] W. K. Hayman, Meromorphic Functions, Oxford University Press, London, 1964. MR 29:1337

[L] B. Q. Li, On normal families in $\mathbf{C}^{n}$, manuscript.

[Mi] C. Miranda, Sur un nouveau critère de normalité pour les familles de fonctions holomorphes, Bull. Soc. Math. France 63 (1935), 185-196.

[Mo] P. Montel, Leçons sur les familles normales des fonctions analytiques et leurs applications, Gauthier-Villars, Paris, 1927.

[N] R. Nevanlinna, Analytic Functions, Springer-Verlag, New York, 1970. MR 43:5003 
[P] X. Pang, Shared values and normal families, Analysis (Munich) 22 (2002), 175-182. MR 2003h:30043

[S] J. L. Schiff, Normal Families, Springer-Verlag, New York, 1993. MR 94f:30046

[Z] L. Zalcman, A heuristic principle in complex function theory, Amer. Math. Monthly 82 (1975), 813-817. MR 52:757

Department of Mathematics, Florida International University, University Park, MiAMI, FLORIDA 33199

E-mail address: libaoqin@fiu.edu 